\section{$\underset{\substack{\text { hommes } \\ \text { \& migrations }}}{ }$}

\section{Hommes \& migrations}

Revue française de référence sur les dynamiques

migratoires

$1301 \mid 2013$

Migrations et mondes ruraux

\title{
Capitalisme agraire et migrations organisées
}

Le cas des plantations de palmier à huile en Indonésie

\section{Stéphanie Barral}

\section{Q OpenEdition}

12 Journals

\section{Édition électronique}

URL : http://journals.openedition.org/hommesmigrations/1926

DOI : 10.4000/hommesmigrations. 1926

ISSN : 2262-3353

Éditeur

Musée national de l'histoire de l'immigration

\section{Édition imprimée}

Date de publication : 1 janvier 2013

Pagination : 119-127

ISBN : 978-2-919040-21-6

ISSN : 1142-852X

\section{Référence électronique}

Stéphanie Barral, « Capitalisme agraire et migrations organisées », Hommes \& migrations [En ligne], 1301 | 2013, mis en ligne le 31 décembre 2015, consulté le 30 avril 2019. URL : http://

journals.openedition.org/hommesmigrations/1926; DOI : 10.4000/hommesmigrations.1926 


\section{CAPITALISME AGRAIRE ET MIGRATIONS ORGANISÉES LE CAS DES PLANTATIONS DE PALMIERS À HUILE EN INDONÉSIE}

par STÉPHANIE BARRAL, docteure en sociologie, chercheure associée CMH-CNRS/EHESS/ENS CIRAD-UMR ART-Dev

En Indonésie, les migrations suivent l'avancée des fronts de colonisation agricole sur la forêt. Les premières régions de grandes plantations de palmiers, situées dans la province de Sumatra Nord, constituent aujourd'hui des zones de départ vers des secteurs nouvellement défrichés. Les migrants qui viennent travailler dans ces plantations parviennent à accéder à la propriété foncière. Avec l'émergence d'un marché du travail et des terres, le front pionnier se développe jusqu'à sa propre saturation qui peut nourrir I'ouverture d'un autre front.

\section{Les premières régions de grandes plantations de palmiers, situées dans la province de Sumatra Nord, constituent aujourd'hui des zones de départ vers des secteurs nouvellement défrichés.}

En Indonésie, les migrations suivent l'avancée des fronts de colonisation agricole sur la forêt. Les premières régions de grandes plantations de palmiers, situées dans la province de Sumatra Nord, constituent aujourd'hui des zones de départ vers des secteurs nouvellement défrichés. Les migrants qui viennent travailler dans ces plantations parviennent à accéder à la propriété foncière. Avec l'émergence d'un marché du travail et des terres, le front pionnier se développe jusquà sa propre saturation qui peut nourrir l'ouverture d'un autre front.
Les grandes plantations constituent la forme historique du capitalisme agraire développée dans la zone intertropicale depuis le XVIe siècle.

Le recours à des travailleurs migrants est une de leurs caractéristiques : l'acheminement d'ouvriers depuis d'autres régions permet à la fois de compenser la présence limitée de travailleurs potentiels au niveau local (du fait de leur présence dans des régions de colonisation agricole récente) et de faciliter le contrôle de ces nouveaux arrivants qui ignorent tout de leur nouvel environnement ${ }^{1}$. 
temporelle des conditions de recrutement de la main-d'œuvre dans les plantations privées en Indonésie : les migrations ouvrières sont déterminées par le fonctionnement des marchés de la terre et du travail en périphérie des plantations et suivent la logique d'évolution d'un front pionnier ${ }^{4}$. Alors que dans les premières années d'installation d'une plantation, les compagnies sont confrontées à la nécessité d'organiser des migrations pour pallier les besoins en main-d'œuvre, la création progressive d'un marché local de la terre entraîne la fixation des migrants et la création d'un marché du travail. Ensuite, quand la colonisation agricole est avancée, les activités économiques atteignent un stade de saturation qui conduit à un renversement du solde migratoire : une région de front pionnier exerce un attrait fort pour les populations migrantes et, à l'inverse, une région entièrement colonisée peut devenir zone de départ, une inversion qui découle d'une certaine stagnation de la croissance économique.

Ainsi, les premières régions de grandes plantations, situées dans la province de Sumatra Nord, sont aujourd'hui des réservoirs de main-d'œuvre migrante partant travailler des régions nouvellement colonisées. Dans

Récolte des régimes de palmier. (c) StÉPHANIE BARRAL

Si de nombreux travaux historiques insistent sur l'importance des migrations dans le développement des plantations ${ }^{2}$, peu de recherches contemporaines sont menées sur le sujet.

Cet article prolonge les travaux de Jan Breman sur lévolution des modes de recrutement en relation avec le contexte économique et les régulations publiques $^{3}$, en proposant une analyse de l'évolution les plantations récentes, les ouvriers connaissent un enrichissement certain par accession à la propriété foncière. Les différentiels d'accumulation entraînent aussi des recompositions notables de lordre social.

L'analyse présentée ici repose sur un double travail. Premièrement, sur une relecture des travaux historiques sur les migrations et les politiques de contrôle de la main-d'œuvre migrante dans les plantations de Sumatra Nord, et, deuxièmement, sur une enquête de terrain d'une année au sein

2. Peter Baak, "The conference on capitalist plantations in colonial Asia, a report", Conference on Capitalist plantations in colonial Asia, Amsterdam, 1990 ; Philipp Curtin, The Rise and Fall of the Plantation Complex. Essays in Atlantic History, Cambridge, Cambridge University Press, 1990. 3. Jan Breman, Labour Migration and Rural Transformation in Colonial Asia, Amsterdam, Free University Press, 1990. 4. Le terme de "front pionnier" désigne le processus de colonisation agricole caractéristique des régions forestières de la zone intertropicale, où une arrivée progressive de populations migrantes défriche et met en valeur progressivement les terres disponibles. Voir à ce sujet François Ruf, Booms et crises du cacao, les vertiges de l'or brun, Paris, ministère de la Coopération, Cirad-Sar et Karthala, 1995 
d'habitations ouvrières dans six grandes plantations indonésiennes. La première partie de l'article retrace l'évolution des modes de migration et de recrutement pendant la période coloniale. Ensuite, c'est dans le contexte de la mise en place de plantations récentes, depuis les années 1980, que ces aspects sont abordés, ainsi que leurs conséquences sur la formation d'un marché du foncier et du travail et sur la structuration d'inégalités au sein du groupe ouvrier. L'analyse de ces processus permet d'appréhender les changements des formes de recrutement et l'évolution actuelle des plantations indonésiennes en fonction des phases successives de colonisation d'un front pionnier.

\section{Les migrations historiques des plantations de Sumatra Nord}

Afin de soutenir le développement agricole, le gouvernement colonial néerlandais vote la Loi agraire de 1870 (Undang-undang Agraria 1870) accordant aux étrangers le droit de contracter des concessions d'une durée de soixante-quinze ans pour des surfaces non utilisées par les populations locales ${ }^{5}$. Cette première étape dans le processus de libéralisation foncière est à l'origine des investissements les plus massifs qu'ait connu la colonie en matière de développement agricole ${ }^{6}$.

La colonisation agricole s'y étend rapidement sur plusieurs centaines de $\mathrm{km}^{2}$, pour l'exploitation desquels le travail de milliers de coolies ${ }^{7}$ est requis. Leur contrôle est réalisé par l'instauration d'un pouvoir disciplinaire qui comprend deux types de domination. Ann Stoler propose une périodisation en deux temps de l'histoire des plantations, fondée sur une dichotomie entre "violence ouverte" (de 1870 aux années 1920) et "violence douce", nouvelle forme de gouvernement des hommes qui apparaît avec la suppression du travail forcé dans les années $1930^{8}$.

Pendant les cinquante premières années du développement des plantations, un régime de travail forcé est à l'œuvre. Les planteurs exploitent le travail des migrants par la force et la coercition. Le recrutement se fait par l'intermédiaire de jobbers qui vont dans les villages javanais promettre fortune aux miséreux. S'installent de réelles relations de patronage et de soumission entre les coolies et le jobber qui exploite leur vulnérabilité et joue le rôle d'intermédiaire avec les dirigeants des plantations.

Bien que le travail forcé soit légalement aboli en 1911, ce régime reste prédominant dans les plantations jusqu'à la fin des années 1920. Les coolies refusant de se soumettre au travail subissent des sanctions (retenues de salaire, heures supplémentaires, corrections physiques ou emprisonnement). Ils vivent entassés dans des baraquements de bois insalubres, surveillés en permanence par des supérieurs hiérarchiques, et peuvent être mutés d'un jour à l'autre dans les diverses divisions des plantations. Une partie de leur salaire est versée en nature, c'est-à-dire en rations de riz. Pour les denrées alimentaires, ils sont dépendants des épiceries des compagnies qui pra-

La volonté de réduire les coûts de recrutement passe par la mise en œuvre effective de l'abolition du travail forcé et l'amélioration des conditions de vie (logement et soins médicaux notamment), mais aussi par l'évolution des modalités du recrutement. tiquent des taux exorbitants. Les maladies bénignes sont soignées par les médecins de la compagnie, et les coolies gravement malades sont renvoyés dans leur village natal. Les planteurs contractualisent les coolies pour des durées de trois à cinq années. Cependant, nombreux sont ceux qui restent ensuite illégalement sur le territoire sumatranais. Progressivement, des villages se constituent dans les périphéries des plantations, et leurs habitants y développent une agriculture vivrière. Ces villages constitueront plus 
tard des réservoirs de main-d'œuvre pour les planteurs cherchant à s'affranchir du recrutement par migration. En 1930, les coolies représentent $30 \%$ de la population totale de l'Oostkust, soit environ un demi-million de personnes ${ }^{9}$.

\section{Du travail forcé au travail libre}

Après cinquante ans de coercition, devant le constat des nombreuses failles des modalités de contrôle des coolies, les planteurs orientent leur politique vers un système de "violence douce", tel que le qualifie Ann Stoler. Les coûts importants de recrutement, les forts taux de mortalité et l'augmentation du nombre de villages illégaux implantés en périphérie de Les ouvriers recrutés plantation sont les premiers surces nouveaux fronts indicateurs de la dégradation de colonisationsont de la situation. Des assauts principalement des migrants.

Contractualisés pour une période de cinq ans, ces jeunes hommes arrivent généralement en famille et s'installent dans les maisons de plantation fournies par la compagnie. contre la hiérarchie sont rapportés régulièrement. Entre 1925 et 1930, l'augmentation des révoltes et des attentats dirigés contre les représentants de l'autorité accélère la prise de conscience de la nécessité de changement. Dès les années 1930, menacés par

les conséquences de la crise économique mondiale de 1929, les planteurs commencent à chercher des éléments de réponse aux révoltes par l'articulation entre travail et vie privée des ouvriers. Le travail libre s'institue progressivement, associé à un encadrement paternaliste plus prononcé.

La volonté de réduire les coûts de recrutement passe par la mise en œuvre effective de l'abolition du travail forcé et l'amélioration des conditions de vie (logement et soins médicaux notamment), mais aussi par l'évolution des modalités du recrutement. Afin de court-circuiter les jobbers, les planteurs recrutent localement ou organisent des migrations en envoyant des coolies en fin de contrat chercher d'autres travailleurs potentiels dans leur village d'origine ${ }^{10}$. La création d'un marché du travail libre confère une plus grande marge de manœuvre aux planteurs quant à la gestion des besoins en travail. Il leur est dorénavant possible d'avoir de la maind'œuvre disponible dans des villages alentour et de la mobiliser en fonction de leurs nécessités. En 1940 , près de $74 \%$ des ouvriers sont recrutés localement ${ }^{11}$. Certains possèdent de petites parcelles de terre propres à l'agriculture vivrière, ce qui limite leur dépendance aux conditions de rémunération par une diversification des activités.

Les conflits sociaux et les mouvements paysans d'occupation illégale des terres ne s'amenuisent pas pour autant, ce qui affaiblit le capitalisme de plantation et limite l'expansion des plantations à la province de Sumatra Nord. Ensuite, l'arrivée au pouvoir d'un régime autoritaire, la mise en place d'une politique nationale de soutien des exportations et le développement d'un modèle de protection sociale légitimant le caractère paternaliste des firmes entraînent un renouveau du modèle de la grande plantation ${ }^{12}$. Depuis quarante ans, les surfaces de grandes plantations ont été multipliées par un facteur 10, couvrant aujourd'hui plus de 4 millions d'hectares sur l'ensemble du territoire indonésien. Les formes des migrations et les politiques de recrutement à l'œuvre y évoluent conjointement avec le développement de dynamiques de front pionnier.

\section{Colonisations récentes et fixation spontanée des populations migrantes}

À partir des années 1970, le développement des plantations de palmiers à huile se déroule progressivement sur le territoire indonésien. Pendant la première décennie, les surfaces mises en valeur 
sont exploitées principalement par des plantations étatiques. Ce n'est qu'à partir des années 1980 que les compagnies privées deviennent l'acteur principal du capitalisme de plantation. L'île de Sumatra est la première cible de cette expansion. Ensuite, c'est à Kalimantan, la partie indonésienne de l'île de Bornéo, que sont développés plusieurs centaines de milliers d'hectares de nouvelles plantations ${ }^{13}$. Depuis les années 2000, ce mouvement d'expansion se poursuit sur ces deux îles et il s'étend aussi à l'Irian Jaya (partie indonésienne de la Papouasie) et aux îles plus petites des archipels des Moluques ou des Célèbes ${ }^{14}$.

Les ouvriers recrutés sur ces nouveaux fronts de colonisation sont principalement des migrants. Contractualisés pour une période de cinq ans, ces jeunes hommes arrivent généralement en famille et s'installent dans les maisons de plantation fournies par la compagnie. La grande majorité d'entre eux sont javanais, mais les dirigeants des compagnies de plantation de Kalimantan recrutent aussi des ouvriers venus d'îles plus proches comme les Célèbes, ou issus de milieux ruraux très pauvres dans les îles orientales de l'archipel.

En Indonésie, les migrations pour le travail, qu'elles soient temporaires ou définitives, sont très fréquentes. Ces dynamiques spontanées, conséquence des inégalités territoriales indonésiennes et des phénomènes de surpopulation et de pauvreté rurales à Java, sont un des facteurs principaux de l'avancée des fronts de colonisation agricole sur la forêt. La dynamique de front pionnier est corrélée à des arrivées massives de migrants venus chercher du travail ou des terres disponibles et faciles d'accès dans ces régions où les services de l'État sont encore absents. Dans le cas des grandes plantations, les migrations organisées ou spontanées sont des moteurs du peuplement.

À la manière des planteurs de l'époque coloniale, les dirigeants des compagnies s'appuient sur les réseaux sociaux des ouvriers et les liens que ces der-

niers entretiennent avec leur village d'origine pour organiser le recrutement: "Avant, au début, jétais au village. Mon village c'est le village de Binjai Baru, à Sumatra Nord. Je suis venu ici [Merantau] à peu près en 1995. Il y avait déjà mes parents ici. Et la première fois que jai rencontré un staff d'ici, en fait la vérité c'est que cette compagnie avait besoin de main-d'œuvre. J'ai connu celui qui s'appelle Pak Sakio, l'assistant de l'estate de M. Il me demande : 'D'où venez-vous? - Je viens du village, je suis venu ici parce que je cherche du travail, je lui réponds. - Là-bas dans votre village vous connaissez beaucoup de monde? - Oui, quand même, il me semble que je peux connaître beaucoup de monde parce que quand même

Petit à petit les migrants renoncent au retour, se rendant compte qu'ils peuvent accéder à la propriété foncière et devenir exploitants agricoles. L'épargne sur salaire permet d'accéder en quelques années à un ou deux hectares de terrain. c'est mon village. - Est-ce que vous pouvez aller chercher de la main-d'ouvre? - Si Dieu veut je pourrais entreprendre cela. [...] C'est comme ça que j’ai ramené six personnes"' (entretien avec un contremaître de plantation de Sumatra, arrivé en 1995 pendant la mise en place de la plantation).

Ce mode de recrutement est cependant lent et coûteux, et les dirigeants organisent aussi des migrations d'une plus grande ampleur. L'île de Java est la cible préférentielle. Le mode de recrutement historique mis en place dès les années 1870 est toujours à l'œuvre, sans que cela entraîne les conflits sociaux qui ont caractérisé les cent premières années de développement des plantations. Un ouvrier ayant été recruté par ce canal explique : "Quand on est arrivé ici, des gens qui étaient arrivés avant, il n'y en avait pas beaucoup. En fait, je suis arrivé avec un groupe de 11 bus qui arrivaient tous depuis Jogja [Java]. Ensuite, il y en a d'autres qui nous ont rejoints, en juillet, puis encore d'autres en novembre, puis encore en décembre. Tous contractualisés à Java. À côté de ça, ils ont aussi fait venir 
de la main-d'ouvre locale. [...] Il y en avait qui arrivaient par leurs propres moyens; il y en avait certains, parce qu'on manquait de monde ici, que nos frères proposaient d'aller chercher dans leurs villages" (entretien avec un ouvrier arrivé en janvier 1989 dans une plantation de Sumatra).

Le statut de salarié représente un réel changement dans la vie des familles ouvrières, qui passent d'un mode de vie reposant sur un futur incertain à la possibilité d'anticiper l'avenir. L'exemple de cet ouvrier récemment embauché est tout à fait parlant à ce sujet : "Je travaille ici depuis... depuis deux ans. Ça ne fait pas longtemps. [...]

Si cela entraîne des dissemblances sociales, c'est parce que tous les ouvriers d'une même plantation ne sont pas recrutés en même temps.

Avec l'augmentation

progressive de la production

et les départs de certains

ouvriers, de nouvelles embauches ont lieu régulièrement.

Avant je travaillais à l'extérieur. Je faisais des petits boulots. J'ai travaillé pendant quatre ans dans la plantation de M. Prapto. Je travaillais et habitais là-bas. Et puis il y a eu des possibilités de rentrer ici et je suis rentré, c'est tout. Dans l'endroit où je travaillais avant, la production commençait à diminuer. Et puis de toute façon quand on travaille pour quelqu'un, on n'a pas de futur. Alors que dans cette plantation on peut penser à notre avenir. Même si c'est pas non plus... en matière d'anticipation, eh bien... on a une retraite ${ }^{15}$. Alors que chez les particuliers, on n'a jamais vu ça. Quand on compte, on est vraiment mieux ici. Je veux dire... le riz, on nous le donne, y a pas besoin d'y penser. On veut aller à l'hôpital, pas besoin d'y penser non plus. C'est ça qui est bien" (entretien avec un ouvrier de plantation de Sumatra).

À leur arrivée, les migrants ont généralement pour objectif de capitaliser quelques années (le temps du contrat de cinq années), puis de rentrer dans leur village d'origine pour y investir les épargnes constituées. Le contexte foncier particulier des fronts pionniers vient bousculer les projets de la grande majorité d'entre eux.

\section{Processus de déprolétarisation des migrants ouvriers}

Petit à petit les migrants renoncent au retour, se rendant compte qu'ils peuvent accéder à la propriété foncière et devenir exploitants agricoles. En effet, contrairement à ce qui est observé des années 1870 aux années 1960, les dirigeants laissent dorénavant la possibilité aux familles ouvrières d'entreprendre librement et de s'enrichir par des investissements personnels. L'épargne sur salaire permet d'accéder en quelques années à un ou deux hectares de terrain. La culture du palmier à huile, connue pour sa forte productivité de la terre, peut entraîner un enrichissement rapide des familles ouvrières. Il se crée dans les régions de front pionnier un marché de la terre et un marché du travail. Les dirigeants s'affranchissent alors des migrations et recrutent les ouvriers localement. Lors des premières années de mise en place d'une plantation, deux phénomènes se conjuguent pour expliquer le démarrage lent des échanges fonciers et de leur monétarisation.

D'une part, à leur arrivée, les migrants ne pensent pas rester dans la région; pour eux, le travail salarié a pour objectif l'épargne en vue d'un retour au village. Ce n'est que progressivement qu'ils défrichent des parcelles de forêt pendant leur temps libre, cultivent du palmier à huile, et projettent leur avenir localement.

D'autre part, les habitants des forêts ont un rapport au foncier très différent des migrants et notamment des Javanais : alors que ces derniers développent une agriculture très intensive sur de petites parcelles, les locaux mettent en valeur la terre par une agriculture de brûlis, itinérante, et une gestion collective et coutumière du foncier. À l'arrivée d'une compagnie, ils n'ont pas conscience du fait que la terre peut avoir une valeur marchande. Les échanges fonciers se font dans un premier temps par du troc et sont peu à peu remplacés par 
des échanges monétaires. C'est ce que met en évidence l'extrait de conversation suivant: "Au début, les Sakai [population locale] ne pensaient pas que leur terre pouvait avoir un prix. Ils en avaient tellement... Alors on pouvait échanger deux hectares contre une radio. Et puis après contre une télé. J'ai même connu quelqu'un qui a échangé dix hectares contre une moto. Ils se sont bien fait avoir. Maintenant ils sont tous partis dans les montagnes. Et maintenant si on veut de la terre, on l'échange avec de l'argent !" (discussion informelle avec un employé de plantation à Sumatra).

Les échanges fonciers timides se transforment progressivement en véritable ruée vers la terre. Au fur et à mesure que les terres sont défrichées et colonisées, le prix du foncier augmente. La colonisation foncière des terres périphériques aux grandes plantations entraîne donc plusieurs phénomènes : elle permet la fixation des travailleurs migrants, ce qui a aussi pour conséquence de structurer un marché local du travail. L'ascension sociale des ouvriers-entrepreneurs ainsi que le développement de l'agriculture paysanne qui en découle créent des besoins en main-d'œuvre dans les petites plantations individuelles, qui peuvent renforcer les migrations spontanées. Petit à petit, les grandes compagnies ont des chances de s'affranchir des migrations organisées.

\section{Avancée d'un front pionnier et différentiels d'accumulation}

La mise en place d'une grande plantation stimule une dynamique de front pionnier, elle-même fondée sur la constitution d'un marché du travail et d'un marché de la terre. Cela entraîne aussi la création d'inégalités d'accès aux moyens de production et donc l'apparition d'une différenciation sociale et économique au sein d'une population migrante initialement marquée par la pauvreté.
Les variations de capital foncier entre ouvriers sont fonction de la durée de l'accumulation économique et foncière qui évolue positivement dans le temps. Si cela entraîne des dissemblances sociales,

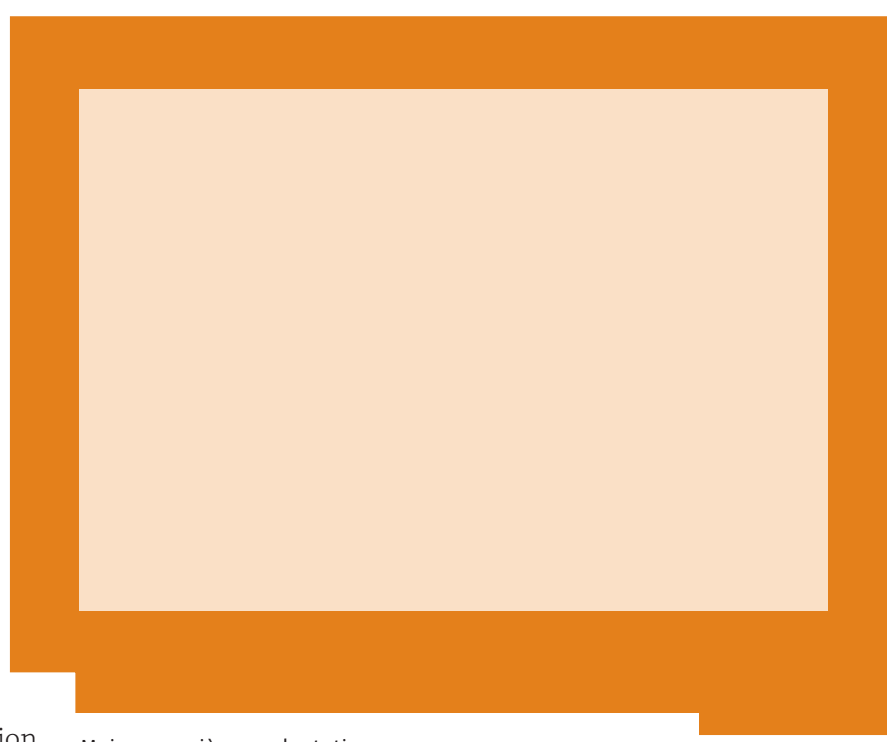

Maison ouvrière en plantation.

(c) Stéphanie Barral

c'est parce que tous les ouvriers d'une même plantation ne sont pas recrutés en même temps. Avec l'augmentation progressive de la production et les départs de certains ouvriers, de nouvelles embauches ont lieu régulièrement. Les ouvriers plus anciennement présents dans une plantation ont donc pu accumuler pendant de plus nombreuses années, et ils possèdent un capital supérieur à celui des nouveaux arrivants. Il y a donc un gradient de répartition des propriétaires fonciers en fonction de la date d'embauche. Cet ouvrier arrivé pendant les premières années de production explique ce phénomène souvent évoqué spontanément lors des entretiens: "Dans cette zone, il y a une partie des ouvriers qui ont de la terre, mais pas tous. Ici, à mon avis, c'est cinquante-cinquante. En fait, ceux qui sont là depuis longtemps, en général ils ont eu le temps d'épargner et d'acheter. Ceux qui sont arrivés récem- 
ment, il y a quelques années seulement, ils n'ont pas encore les moyens. C'est commeça" (entretien avec un ouvrier de plantation à Sumatra).

La capacité d'accumulation de chaque famille n'évolue cependant pas de manière strictement linéaire dans le temps. En effet, le prix du foncier augmente progressivement avec la colonisation agricole. Le nombre d'années d'épargne nécessaire à l'achat d'une parcelle

Ce processus a pour conséquence d'alimenter le marché du travail localement puisque la culture du palmier à huile, connue pour sa haute productivité de la terre, permet de recourir à des travailleurs salariés croît donc en conséquence. Une famille arrivée pendant les premières années de mise en place ou de production d'une plantation peut accéder à du foncier en quelques mois. À l'inverse, après une vingtaine d'années de désenclavement et de défriche, plusieurs années d'épargne sont nécessaires pour acquérir une parcelle. Par exemple, les premiers arrivants d'une plantation étudiée dans la province de Riau (Sumatra) troquaient la terre, puis étaient capables d'acheter deux hectares après une ou deux années de travail. Il leur fallait ensuite encore quelques années d'épargne et de travail pour défricher et installer une plantation de palmiers. À l'heure actuelle, la durée moyenne d'épargne pour acheter deux hectares y est d'une dizaine d'années.

Dans les plantations se côtoient ainsi des ouvriers possédant une dizaine d'hectares et parfois même une voiture personnelle, et d'autres n'ayant encore d'autre source de revenu que leur salaire de récolteur. La similitude sociale caractérisant la phase initiale de constitution de la communauté ouvrière, lors des premiers temps de mise en place d'une plantation, fait progressivement place à une différenciation économique des revenus des familles et donc à une dissemblance économique prononcée.

Ce processus a pour conséquence d'alimenter le marché du travail localement puisque la culture du palmier à huile, connue pour sa haute productivité de la terre, permet de recourir à des travailleurs salariés : les ouvriers qui possèdent quatre hectares ou plus emploient généralement de la maind'œuvre intérimaire pour récolter les régimes de leurs plantations. L'attractivité de la région en est augmentée et le marché du travail se structure localement autour des emplois d'ouvriers permanents dans les grandes plantations et des emplois temporaires chez les petits planteurs. Ainsi, les grandes compagnies peuvent s'affranchir de l'organisation de recrutements lointains. Alors que dans les premières années de désenclavement l'accès à la maind'œuvre est problématique, il est facilité après une dizaine d'années de production.

\section{Marché du foncier démarchandisé et migrations circulaires}

Les mécanismes de front pionnier ne s'opèrent pas de manière si simple partout. Et le degré de marchandisation de la terre n'est pas le même dans toutes les régions : le rapport que les populations autochtones ont à la coutume peut entraîner une certaine démarchandisation. Dans le cas d'une plantation étudiée à Sumatra, le chef d'un groupe social situé au nord de la plantation refuse catégoriquement que des parcelles soient vendues à des migrants, alors que dans le sud de la plantation, les locaux, beaucoup plus pauvres, vendent facilement leur terrain. Dans une autre plantation à Kalimantan, l'enquête montre que la majorité des migrants interrogés possédant de la terre y ont eu accès en se mariant avec une personne du groupe social local. Cela traduit une forme de démarchandisation de la terre, induite par une certaine résistance des communautés locales à échanger le foncier. Dans d'autres cas, les populations locales ont choisi la fuite dans des terres plus reculées. L'accès au foncier est donc variable, ce qui signifie que le phénomène d'ascension sociale analysé ici évolue non seulement dans le temps, mais aussi qu'il ne présente pas la même intensité dans toutes les plantations. Cela 
a des conséquences directes sur la structuration locale des marchés de la terre et du travail, qui déterminent eux-mêmes les politiques de recrutement et de migration mises en œuvre par les compagnies de plantations. En effet, les migrants ne pouvant avoir accès à la terre ne se fixent pas localement. Ils mettent en œuvre des stratégies d'épargne destinées à investir dans leur village d'origine. Les migrations sont alors de type circulaire : après quelques années de travail, les hommes venus seuls travailler, ou les familles entières qui ont fait le déplacement, retournent à leurs parcelles ou à leurs activités laissées dans les zones rurales qu'ils ont quittées. C'est le cas de la plantation enquêtée sur Kalimantan. Ce phénomène est aussi mis en évidence dans des études de villages de départ des migrations sur lî̀le de Lombok. Ces migrations circulaires sont cependant plus fréquentes dans les plantations malaisiennes où des migrants indonésiens partent chercher des salaires plus élevés que dans leur pays ${ }^{16}$.

\section{Conclusion}

Alors qu'historiquement les migrations organisées de Java à Sumatra Nord ont été l'occasion de conflits sociaux forts, les recrutements d'ouvriers de plantation sont aujourd'hui caractérisés par une paix sociale notable. La non-effectivité des régulations publiques de la terre entraîne un mouvement de fixation des migrants par accession à la propriété foncière et donc une ascension sociale pronon- cée de ces populations. Cela a pour conséquence l'affranchissement progressif par les compagnies de l'organisation de migrations depuis d'autres îles ou d'autres régions du pays.

Cependant, un point noir se profile à l'horizon de cette configuration. En effet, l'analyse de la condition ouvrière des compagnies de Sumatra Nord, berceau historique des plantations en Indonésie, montre que les régions anciennement colonisées, où il ne reste plus de terres vierges à mettre en culture, connaissent des densités de population fortes et des taux d'inactivité et de chômage importants. Les phénomènes d'accumulation économique sont limités et les ouvriers de plantation ne connaissent pas les trajectoires d'ascension sociale observées dans les plantations récentes. Ces régions ne sont plus des bassins d'emploi et d'activité attractifs, bien au contraire : la saturation foncière et économique a entrainé un renversement de situation, et nombreux sont les hommes à partir tenter leur chance dans d'autres régions du pays. Ces migrants ruraux alimentent notamment les besoins en main-d'œuvre des plantations récentes mises en place dans le reste du pays. Les dynamiques actuelles peuvent donc cacher des phénomènes d'appauvrissement futurs tels qu'observés à Sumatra Nord, conséquence de la consommation de la ressource forestière. Si les dirigeants des plantations ont pu s'affranchir des migrations, ils pourront aussi faire face à de nouvelles problématiques sociales liées aux difficultés économiques régionales. 